\title{
THE DECAY OF SOLUTIONS OF THE TWO DIMENSIONAL WAVE EQUATION IN THE EXTERIOR OF A STRAIGHT STRIP
}

BY

\author{
PETER WOLFE $(1)$
}

\begin{abstract}
ABSTRACr. We study an initial boundary value problem for the wave equation in the exterior of a straight strip. We assume the initial data has compact support and that the solution vanishes on the strip. We then show that at any point in space the solution is $O(1 / t)$ as $t \rightarrow \infty$. This is the same rate of decay as obtains for the solution of the initial boundary value problem posed in the exterior of a smooth star shaped region. Our method is to use a Laplace transform. This reduces the problem to a consideration of a boundary value problem for the Helmholtz equation. We derive estimates for the solution of the Helmholtz equation for both high and low frequencies which enable us to obtain our results by estimating the Laplace inversion integral asymptotically.
\end{abstract}

0. Introduction. We consider an initial boundary value problem for the two dimensional wave equation in the exterior of a straight strip. We assume that the initial data have compact support and that the solution vanishes on the strip. Our object is to obtain the rate of decay of the solution to this problem as $t \rightarrow \infty$. In a recent paper [10] we considered the problem of diffraction of a plane pulse by a strip. Here we shall proceed much as in [10] and will use some results from that paper. We use the method of Laplace transformation. Our results are obtained by evaluating the inversion integral asymptotically. We show that the contour of the inversion integral can be deformed into the left half plane. Then by considering separately the integrals over the portions of the contour near the origin and far away from it we show that the solution decays at any point in space like $1 / t$.

Most of the work of the paper goes into proving estimates for solutions of the reduced wave equation. To obtain these estimates we study the integral equation

Received by the editors June 2, 1975 and, in revised form, February 3, 1976.

AMS (MOS) subject classifications (1970). Primary 35L05; Secondary 35A22, 35B40, 35C10.

Key words and phrases. Wave equation, rate of decay of solutions, asymptotic behavior of solutions, Laplace transform, reduced wave equation.

(1) This research was supported in part by the National Science Foundation under Grant GP 37753.

- American Mathematical Society 1977 


$$
\begin{aligned}
& -H_{0}^{(1)}\left(\kappa\left[(s-x)^{2}+y^{2}\right]^{1 / 2}\right) \\
& \quad=\int_{-1}^{1} \tilde{\rho}\left(s^{\prime}, x, y, \kappa\right) H_{0}^{(1)}\left(\kappa\left|s-s^{\prime}\right|\right) d s^{\prime}, \quad|s| \leqslant 1 .
\end{aligned}
$$

Two cases must be considered. First we treat the "high frequency" case in which $\kappa=\kappa_{1}+i \kappa_{2}$ with $\kappa_{1} \gg 0$ and $\kappa_{2} \leqslant 0$. Here we use a modification of the technique developed in [8] and [9] and used in [10]. We must also consider the "low frequency" case: $\kappa$ small and positive. Here we use the method of Sologub [6]. However we find it desirable to recast his method in terms of function spaces previously introduced by the author in order to obtain useful estimates.

In [3] it was shown that the solution of the initial boundary value problem in the exterior of a smooth star shaped region decays like $1 / t$. (It is now known that in three dimensions the solution decays exponentially [2]. Although in [3] the author considers the three dimensional case she remarks that the result is also true in two dimensions. See also [4] for a related result.) The result is obtained in [3] by using an energy integral technique (i.e., clever use of the divergence theorem). So far attempts to apply this method to the present problem have been unsuccessful. One basic difficulty is that the derivatives of the solution are unbounded near the edge of the strip. Also the (one sided) normal derivative of $U$ is not square integrable over the strip. Thus we proceed here in a different manner.

Our result is of interest for it seems to show that the phenomenon of edge diffraction which is present here does not play a significant role in energy decay.

The plan of the paper is as follows: In $\S 1$ we will obtain the decay theorem modulo the results on the asymptotic behaviour of the solution of $(0.1)$. In $\$ 2$ we will derive the high frequency result while in $\$ 3$ we will derive the low frequency result.

1. The theorem on the rate of decay. We consider here the wave equation in two spaces variables

$$
V_{x x}+V_{y y}=V_{t t}
$$

We look for a solution $V(x, y, t)$ of (1.1) for $t>0$ which vanishes on the strip $y=0,|x| \leqslant 1$. The initial conditions are

$$
V(x, y, 0)=f(x, y), \quad V_{t}(x, y, 0)=g(x, y) .
$$

We assume that $f \in C^{3}\left(R^{2}\right), g \in C^{2}\left(R^{2}\right)$ and that the supports of $f$ and $g$ are contained in a compact set $K$ which does not meet the strip.

The free space solution to the problem (1.1), (1.2) is given by 


$$
\begin{aligned}
W(x, y, t)= & \frac{1}{2 \pi} \iint \frac{H\left(t-\left(x^{\prime 2}+y^{\prime 2}\right)^{1 / 2}\right) g\left(x+x^{\prime}, y+y^{\prime}\right)}{\left(t^{2}-x^{\prime 2}-y^{\prime 2}\right)^{1 / 2}} d x^{\prime} d y^{\prime} \\
& +\frac{1}{2 \pi} \frac{\partial}{\partial t} \iint \frac{H\left(t-\left(x^{\prime 2}+y^{\prime 2}\right)^{1 / 2}\right) f\left(x+x^{\prime}, y+y^{\prime}\right)}{\left(t^{2}-x^{\prime 2}-y^{\prime 2}\right)^{1 / 2}} d x^{\prime} d y^{\prime} .
\end{aligned}
$$

In (1.3) $H(x)$ is the Heaviside function and the integration is taken over the whole $\left(x^{\prime}, y^{\prime}\right)$ plane. We let $U=V-W$. Then $U$ will satisfy (1.1) and have the initial and boundary values

$$
\begin{aligned}
& U(x, y, 0)=U_{t}(x, y, 0)=0, \\
& U(x, 0, t)=-W(x, 0, t), \quad t>0,|x| \leqslant 1 .
\end{aligned}
$$

We will construct $U$ as follows: we formally apply a Laplace transform to (1.1) (for $U$ ) and (1.4). If we define $u(x, y, \sigma)$ to be the (formal) Laplace transform of $U(x, y, t)$,

$$
u(x, y, \sigma)=\int_{0}^{\infty} e^{-\sigma t} U(x, y, t) d t
$$

then $u$ must satisfy the reduced wave equation

$$
u_{x x}+u_{y y}-\sigma^{2} u=0
$$

with the boundary condition

$$
u(x, 0, \sigma)=-w(x, 0, \sigma), \quad|x| \leqslant 1,
$$

where $\omega(x, y, \sigma)$ is the Laplace transform of $W$. The function $w$ is given by

$$
\begin{aligned}
w(x, y, \sigma)= & \iint g\left(x+x^{\prime}, y+y^{\prime}\right) K_{0}\left(\sigma\left[x^{\prime 2}+y^{\prime 2}\right]^{1 / 2}\right) d x^{\prime} d y^{\prime} \\
& +\sigma \iint f\left(x+x^{\prime}, y+y^{\prime}\right) K_{0}\left(\sigma\left[x^{\prime 2}+y^{\prime 2}\right]^{1 / 2}\right) d x^{\prime} d y^{\prime} .
\end{aligned}
$$

Hence

$$
\begin{aligned}
w(x, 0, \sigma)= & \iint_{K} g(\xi, \eta) K_{0}\left(\sigma\left[(x-\xi)^{2}+\eta^{2}\right]^{1 / 2}\right) d \xi d \eta \\
& +\sigma \iint_{K} f(\xi, \eta) K_{0}\left(\sigma\left[(x-\xi)^{2}+\eta^{2}\right]^{1 / 2}\right) d \xi d \eta .
\end{aligned}
$$

Here $K_{0}$ denotes Macdonald's Bessel function of order zero. In addition, $u(x, y, \sigma)$ is required to be analytic for $\operatorname{Re} \sigma>0$ and $u(x, y, \sigma) \rightarrow 0$ as $\operatorname{Re} \sigma$ $\rightarrow \infty$.

We will prove existence of $u$ in a region containing the right half plane 
(Theorem $\left.2^{\prime}\right)$. The properties of $u$ will insure that if we define $U(x, y, t)$ by the Laplace inversion integral taken along the imaginary axis in the $\sigma$-plane,

$$
U(x, y, t)=\frac{1}{2 \pi} \int_{-\infty}^{\infty} e^{i \sigma_{2} t} u\left(x, y, i \sigma_{2}\right) d \sigma_{2}
$$

then $U$ will be the solution of (1.1), (1.4).

The solution we seek of (1.5) and (1.6) is given by

$$
u(x, y, \sigma)=\int_{-1}^{1} \rho(s, x, y, \sigma) w(s, 0, \sigma) d s .
$$

The function $\rho(s, x, y, \sigma)$ represents the jump in the normal derivative of the Green's function for the problem across the strip [9]. It is the solution of the integral equation

$$
\text { (1.9) }-K_{0}\left(\sigma\left[(s-x)^{2}+y^{2}\right]^{1 / 2}\right)=\int_{-1}^{1} \rho\left(s^{\prime}, x, y, \sigma\right) K_{0}\left(\sigma\left|s-s^{\prime}\right|\right) d s^{\prime}, \quad|s| \leqslant 1 .
$$

Reasoning as in [10] we see that (1.9) admits a unique solution $\rho(s, x, y, \sigma)$ for every $(x, y)$ not on the strip and every $\sigma \neq 0$ with $\operatorname{Re} \sigma \geqslant 0$ such that when this $\rho$ is inserted in (1.8) the function $u$ thus defined is a solution of (1.5) and (1.6) which is analytic for $\operatorname{Re} \sigma>0$.

We wish to show that $u(x, y, \sigma)$ can be analytically continued into the left half plane. We first consider $w(s, 0, \sigma)$ given by (1.7). This will be an analytic function of $\sigma$ in the $\sigma$-plane slit along the negative real axis.

LEMMA 1.1. If $\sigma_{1}=\operatorname{Re} \sigma \leqslant 0$, then for some positive constants $C$ and $B$,

$$
|w(s, 0, \sigma)| \leqslant C|\sigma|^{-5 / 2} e^{-\sigma_{1} B}, \quad|s| \leqslant 1, \pi / 2<|\arg \sigma|<\pi .
$$

Proof. Since $K$ is compact and does not meet the strip we can cover $K$ with circles $\left\{C_{i}\right\}_{i=1}^{n}$ such that for $(\xi, \eta) \in C_{i}$ either (a) $|\eta| \geqslant \eta_{0}$, (b) $\xi-1 \geqslant \delta>0$, or (c) $-1-\xi \geqslant \delta>0$. Let $\left\{\theta_{i}\right\}_{i=1}^{n}$ be a family of $C^{\infty}$ functions such that the support of each $\theta_{i}$ is contained in $C_{i}$ and $\sum_{i=1}^{n} \theta_{i}=1$ on $K$. Let $f_{i}=\theta_{i} f, g_{i}$ $=\theta_{i} g$.

Now consider for instance

$$
\iint_{C_{i}} g_{i}(\xi, \eta) K_{0}\left(\sigma\left[(s-\xi)^{2}+\eta^{2}\right]^{1 / 2}\right) d \xi d \eta
$$

where on $C_{i},|\eta| \geqslant \eta_{0}$. Since $\left[(s-\xi)^{2}+\eta^{2}\right]$ is bounded away from zero uniformly for $|s| \leqslant 1$ and $(\xi, \eta) \in K$, we may replace $K_{0}$ by its asymptotic expansion 


$$
K_{0}(\sigma R)=(2 / \pi \sigma R)^{1 / 2} e^{-\sigma R}[1-1 / 8 \sigma R+\varepsilon(\sigma R)]
$$

where $|\varepsilon(z)| \leqslant C_{3} /|z|^{2}$.

Equation (1.11) is valid for $|\arg \sigma|<\pi$. In (1.11) we have set

$$
R=\left[(s-\xi)^{2}+\eta^{2}\right]^{1 / 2} .
$$

Let us estimate

$$
\frac{1}{\sigma^{1 / 2}} \iint_{C_{i}} \frac{e^{-\sigma R}}{R^{1 / 2}} g_{i}(\xi, \eta) d \xi d \eta
$$

Since $R_{\eta}=\eta / R$ this is equal to

$$
\begin{aligned}
-\frac{1}{\sigma^{3 / 2}} \iint_{C_{i}} e^{-\sigma R}\left(-\sigma R_{\eta}\right) \frac{R^{1 / 2}}{\eta} g_{i}(\xi, \eta) d \xi d \eta \\
=\frac{1}{\sigma^{3 / 2}} \iint_{C_{i}} e^{-\sigma R}\left(\frac{R^{1 / 2}}{\eta} g_{i}(\xi, \eta)\right)_{\eta} d \xi d \eta .
\end{aligned}
$$

Here we use the fact that in $C_{i},|\eta| \geqslant \eta_{0}$. A second integration by parts shows that this term satisfies an estimate of the form (1.10) where $B$ $=\max _{|s| \leqslant 1 ;(\xi, \eta) \in C_{i}} R$. The term

$$
\frac{1}{\sigma^{3 / 2}} \iint_{C_{i}} \frac{e^{-\sigma R}}{R^{3 / 2}} g_{i}(\xi, \eta) d \xi d \eta
$$

is handled similarly (one integration by parts) while the remainder term clearly yields the estimate (1.10). The integral involving $f$ is handled in the same way. If in $C_{i}, \xi-1 \geqslant \delta$ or $-\xi-1 \geqslant \delta$, the integrations by parts are carried out with respect to $\xi$. This completes the proof of the lemma.

We now consider the function $\rho(s, x, y, \sigma)$. Letting $\kappa=i \sigma$ transforms (1.9) into equation (0.1) with $\tilde{\rho}(s, x, y, \kappa)=\rho(s, x, y,-i \kappa)$. We wish to continue $\rho$ into the left half $\sigma$-plane. In $\$ 2$ we consider $(0.1)$ for $\kappa=\kappa_{1}+i \kappa_{2}, \kappa_{1}>0, \kappa_{2} \leqslant 0$. This corresponds to $\sigma=\sigma_{1}+i \sigma_{2}$ with $\sigma_{1} \leqslant 0, \sigma_{2}<0$, i.e., $\sigma$ in the third quadrant. But since $K_{0}(\sigma R)$ is real for real $\sigma$ we see from (1.9) that if $\operatorname{Re} \sigma>0$,

$$
\rho(s, x, y, \bar{\sigma})=\overline{\rho(s, x, y, \sigma)} .
$$

Thus we may extend our results to the case where $\sigma$ is in the second quadrant by reflection. Hence we have from Theorem 2 of $\$ 2$

THEOREM $2^{\prime}$. Equation (1.9) admits a unique solution $\rho(s, x, y, \sigma)$ for $\sigma$ satisfying 


$$
e^{-\sigma_{1}}<4 \pi^{1 / 4}\left|\sigma_{2}\right|^{3 / 4}, \quad \sigma_{1} \leqslant 0,
$$

such that if $u(x, y, \sigma)$ is given by (1.8) $u$ is analytic in $\sigma$ for $\sigma$ in the union of the regions given by (1.12a) and the half plane $\sigma_{1}>0$. If

$$
e^{-\sigma_{1}} \leqslant 8^{1 / 2} \pi^{1 / 4}\left|\sigma_{2}\right|^{3 / 4}, \quad \sigma_{1} \leqslant 0 .
$$

Then $\rho$ satisfies the estimate (cf. (2.10))

$$
\begin{aligned}
|\rho(s, x, y, \sigma)| \leqslant & \frac{4}{\pi}\left|\frac{\partial}{\partial r} K_{0}\left(\sigma\left[(x-s)^{2}+(y-r)^{2}\right]^{1 / 2}\right)\right|_{r=0} \mid \\
& +C\left|\sigma_{2}\right|^{-1 / 2}\left(1+\left|\sigma_{2}\right|^{-1 / 2}\right) e^{-\sigma_{1}(|x|+|y|+1)} \\
& \times\left(e^{\sigma_{1}(s-1)}(1-s)^{-1 / 2}+e^{-\sigma_{1}(s+1)}(1+s)^{-1 / 2}\right) .
\end{aligned}
$$

The proof of analyticity is exactly the same as in [10]. By (1.8), (1.10) and (1.13) we find that if (1.12b) holds

$$
|u(x, y, \sigma)| \leqslant C|\sigma|^{-2} e^{-D \sigma_{1}} .
$$

The quantities $C$ and $D$ in (1.14) depend on $x$ and $y$, but (with a little more work in \$2) the estimate (1.14) can be made uniform over compact subsets of the plane with the closed strip removed.

The solution of (1.1) (for $U$ ) and (1.4) is defined by

$$
U(x, y, t)=\frac{1}{2 \pi i} \int_{\Gamma} e^{\sigma t} u(x, y, \sigma) d \sigma .
$$

The contour $\Gamma$ can be taken to be the imaginary axis (as we shall see $u \sim \log |\sigma|$ near $\sigma=0$ ).

Let $\Gamma^{\prime}$ be the contour $\Gamma_{1} \cup \Gamma_{2} \cup \Gamma_{3}$ where

$$
\begin{aligned}
& \Gamma_{1}: e^{-\sigma_{1}}=8^{1 / 2} \pi^{1 / 4}\left(-\sigma_{2}\right)^{3 / 4}, \sigma_{1}<0, \\
& \Gamma_{2}: \sigma_{1}=0,-1 / 4 \pi^{1 / 3} \leqslant \sigma_{2} \leqslant 1 / 4 \pi^{1 / 3}, \\
& \Gamma_{3}: e^{-\sigma_{1}}=8^{1 / 2} \pi^{1 / 4} \sigma_{2}^{3 / 4}, \sigma_{1}<0 .
\end{aligned}
$$

$\Gamma^{\prime}$ is oriented so that $\sigma_{2}$ is increasing (Figure 4).

We wish to show that the integral in (1.15) can be taken along $\Gamma^{\prime}$ for sufficiently large $t$. For this it is sufficient to show that the integral along a horizontal segment between $\Gamma$ and $\Gamma^{\prime}$ tends to zero as $\left|\sigma_{2}\right| \rightarrow \infty$.

Thus let

$$
I_{\sigma_{2}}=\int_{\sigma_{1}^{*}}^{0} e^{\sigma t} u(x, y, \sigma) d \sigma_{1}
$$

where $e^{-\sigma_{1}^{*}}=8^{1 / 2} \pi^{1 / 4}\left|\sigma_{2}\right|^{3 / 4}$. By (1.14) if $t>D$, 


$$
\left|I_{\sigma_{2}}\right| \leqslant \frac{C}{\left|\sigma_{2}\right|^{2}} \int_{\sigma_{1}^{*}}^{0} e^{\sigma_{1}(t-D)} d \sigma_{1}=\frac{C}{\left|\sigma_{2}\right|^{2}} \frac{1-e^{\sigma_{1}^{*}(t-D)}}{t-D} \leqslant \frac{C}{\left|\sigma_{2}\right|^{2}} \frac{1}{t-D} .
$$

Thus, if $t>D,\left|I_{\sigma_{2}}\right| \rightarrow 0$ as $\left|\sigma_{2}\right| \rightarrow \infty$ and we have

$$
U(x, y, t)=\frac{1}{2 \pi i} \int_{\Gamma^{\prime}} e^{\sigma t} u(x, y, \sigma) d \sigma .
$$

We next show

$$
\int_{\Gamma_{i}} e^{\sigma t} u(x, y, \sigma) d \sigma=O\left(t^{-1}\right) \quad \text { as } t \rightarrow \infty, \quad i=1,3 .
$$

To prove (1.17) we parameterize $\Gamma_{3}$ by $\sigma_{2}$. On $\Gamma_{3}, e^{-\sigma_{1}}=8^{1 / 2} \pi^{1 / 4} \sigma_{2}^{3 / 4}, d \sigma_{1}$ $=-(3 / 4)\left(d \sigma_{2} / \sigma_{2}\right)$ and $|d \sigma| \leqslant C d \sigma_{2}$. By $(1.14)$

$$
\begin{aligned}
\left|\int_{\Gamma_{3}} e^{\sigma t} u(x, y, \sigma) d \sigma\right| & \leqslant C \int_{\left(4 \pi^{1 / 3}\right)^{-1}}^{\infty} e^{-\sigma_{1}(D-t)} \sigma_{2}^{-2} d \sigma_{2} \\
& =C(64 \pi)^{(D-t) / 4} \int_{\left(4 \pi^{1 / 3}\right)^{-1}}^{\infty} \sigma_{2}^{\frac{3}{4}(D-t)-2} d \sigma_{2} \\
& =4 \pi^{1 / 3} C\left[1+\frac{3}{4}(t-D)\right]^{-1}=O(1 / t) .
\end{aligned}
$$

The integral over $\Gamma_{1}$ is treated similarly.

Setting $a=\left(4 \pi^{1 / 3}\right)^{-1}$ we now have

$$
U(x, y, t)=\frac{1}{2 \pi} \int_{-a}^{a} e^{i \sigma_{2} t} u\left(x, y, i \sigma_{2}\right) d \sigma_{2}+O(1 / t)
$$

We now consider the behavior of $u\left(x, y, i \sigma_{2}\right)$ near $\sigma=0$. From (3.41) we have

$$
\begin{aligned}
& \rho(s, x, y,-i \kappa)=(-1 / \pi) T\left(R^{\prime} / R\right)(s) \\
& \quad+\frac{1}{4 \ln (\kappa \gamma / 4 i)}\left[-\ln \frac{\kappa \gamma}{2 i} R_{0}+\frac{1}{\pi} \int_{-1}^{1} \ln |t| T\left(\frac{R^{\prime}}{R}\right)(t) d t\right]\left(1-s^{2}\right)^{-1 / 2} \\
& +\rho_{1}(s, x, y, \kappa), \quad \kappa>0 .
\end{aligned}
$$

In (1.19) $R=\left((x-s)^{2}+y^{2}\right)^{1 / 2}, R^{\prime}=\partial R / \partial s, R_{0}=\left(x^{2}+y^{2}\right)^{1 / 2}$. The operator $T$ is defined by (3.7), but for the present purposes it is enough to note that $T\left(R^{\prime} / R\right) \in L_{1}[-1,1]$ and the integral in (1.19) is finite. The remainder term $\rho_{1}(s, x, y, \kappa)$ satisfies

$$
\left\|\rho_{1}\right\|_{L_{1}}=O\left(\kappa^{2} \ln \kappa\right), \quad\left\|\partial \rho_{1} / \partial \kappa\right\|_{L_{1}}=O(\kappa \ln \kappa), \quad \kappa>0
$$

Also in (1.23) $\ln (\kappa \gamma / 4 i)=\ln (\sigma \gamma / 4)$, where $\sigma=-i \kappa, \ln \gamma=0.57721 \cdots$ (Euler's constant) and the logarithm is taken to be real for $\sigma>0$. With this 
definition of the logarithm, (1.19) and (1.20) hold also for $\kappa<0$.

From (1.7), using the series expansion for $K_{0}$, we find

$$
w(s, 0, \sigma)=a(s) \ln (\sigma \gamma / 2)+b(s, \sigma)
$$

where $a(s)$ and $b(s)$ are continuous in $s$ for $|s| \leqslant 1$ and

$$
|b(s, \sigma)| \leqslant C|\sigma \ln \sigma|, \quad\left|\frac{\partial b}{\partial \sigma}(s, \sigma)\right| \leqslant C|\ln \sigma|, \quad|s| \leqslant 1 .
$$

Using (1.19) and (1.21) in (1.8) we find for $\sigma=-i \kappa$,

$$
u(x, y, \sigma)=\alpha(x, y) \ln \frac{\sigma \gamma}{4}+\beta(x, y)+\delta(x, y)\left(\ln \frac{\sigma \gamma}{4}\right)^{-1}+\varepsilon(x, y, \sigma)
$$

where

$$
|\varepsilon| \leqslant C|\sigma \ln \sigma|, \quad|\partial \varepsilon / \partial \sigma| \leqslant C|\ln \sigma| .
$$

In (1.23) $\alpha, \beta, \delta, \varepsilon$ are bounded in $(x, y)$ uniformly for $(x, y)$ in compact subsets of the plane with the strip removed.

From (1.18) and (1.23),

$$
\begin{aligned}
U(x, y, t)=\frac{1}{2 \pi} \int_{-a}^{a} e^{i \sigma_{2} t}[ & \alpha(x, y) \ln (\sigma \gamma / 4)+\beta(x, y) \\
& \left.+\delta(x, y)(\ln (\sigma \gamma / 4))^{-1}+\varepsilon(x, y, \sigma)\right] d \sigma_{2}+O(1 / t) .
\end{aligned}
$$

Using (1.24) we see by integrating by parts that the term involving $\varepsilon$ is $o(1 / t)$. Clearly the term involving $\beta$ is $O(1 / t)$. Also if $\Gamma_{ \pm}$are the half lines running from $-\infty \pm i a$ to $\pm i a$, then

$$
\int_{\Gamma_{ \pm}} e^{\sigma t}\left(\alpha(x, y) \ln \frac{\sigma \gamma}{4}+\delta(x, y)\left(\ln \frac{\sigma \gamma}{4}\right)^{-1}\right) d \sigma=o\left(\frac{1}{t}\right)
$$

Hence

$$
U(x, y, t)=\frac{1}{2 \pi i} \int_{\gamma_{0}} e^{\sigma t}\left(\alpha(x, y) \ln \frac{\sigma \gamma}{4}+\delta(x, y)\left(\ln \frac{\sigma \gamma}{4}\right)^{-1}\right) d \sigma+o\left(\frac{1}{t}\right)
$$

where $\gamma_{0}$ is a contour starting at $-\infty-i$, circling the origin and ending at $-\infty+i$. The negative real axis is a branch cut for $\ln \sigma$. We have

$$
\frac{1}{2 \pi i} \int_{\gamma_{0}} e^{\sigma t} \ln \sigma d \sigma=-\int_{-\infty}^{0} e^{\sigma_{1} t} d \sigma_{1}=-\frac{1}{t}
$$


Finally, reasoning as in [10], we see that

$$
\int_{\gamma_{0}} e^{\sigma t} \frac{d \sigma}{(\ln (\sigma \gamma / 4))}=O\left(\frac{1}{t \ln (4 t / \gamma)}\right)
$$

Thus $U(x, y, t)=O(1 / t)$.

The solution to our problem $V$ is given by $V=U+W$ where $W$ given by (1.3) is the free space solution of the problem (1.1), (1.2). Since $W(x, y, t)$ $=O(1 / t)$ we have proven

THEOREM 1. Let $V$ be the solution of (1.1), (1.2) which vanishes on the strip $\{(x, y): y=0,|x| \leqslant 1\}$. Then at any point in space $V$ decays like $1 / t$.

REMARK. It can be shown that the decay rate is uniform on compact subsets of the plane with the strip removed.

2. Solution of the integral equation in the "high frequency" case. In this section we study the integral equation

(2.1) $-H_{0}^{(1)}\left(\kappa\left[\left(x^{\prime}-x\right)^{2}+y^{2}\right]^{1 / 2}\right)=\int_{-1}^{1} \rho(\xi) H_{0}^{(1)}\left(\kappa\left|\xi-x^{\prime}\right|\right) d \xi, \quad\left|x^{\prime}\right| \leqslant 1$.

We have written $\tilde{\rho}(\xi, x, y, \kappa)=\rho(\xi)$ for brevity. We assume $\kappa=\kappa_{1}+i \kappa_{2}, \kappa_{1}$ $>0, \kappa_{2} \leqslant 0$. We look for a solution of $(2.1)$ of the form

(2.2) $\rho(\xi)=\frac{1}{4 \pi} \int_{\gamma}\left(\alpha^{2}-\kappa^{2}\right)^{-1 / 2} \rho(\xi, \alpha) \exp \left\{i \alpha x-\left(\alpha^{2}-\kappa^{2}\right)^{1 / 2}|y|\right\} d \alpha$.

The choice of contour $\gamma$ depends on the position of $(x, y)$. The function $\rho(\xi, \alpha)$ is given by

$$
\begin{aligned}
\rho(\xi, \alpha)= & -2\left(\alpha^{2}-\kappa^{2}\right)^{1 / 2} e^{-i \alpha \xi}+\int_{C_{1}} e^{i k(\xi-1)} \phi_{1}(k, \alpha) d k \\
& +\int_{C_{2}} e^{i k(\xi+1)} \phi_{2}(k, \alpha) d k .
\end{aligned}
$$

The functions $\phi_{1}$ and $\phi_{2}$ are assumed to satisfy the pair of integral equations

$$
\begin{array}{r}
\pi i X_{1}^{+}(k) \phi_{1}(k, \alpha)=\frac{2(-\alpha-\kappa)^{1 / 2} e^{-i \alpha}}{\alpha+k}+\int_{C_{2}} \frac{\phi_{2}\left(k^{\prime}, \alpha\right) X_{1}\left(k^{\prime}\right) e^{2 i k^{\prime}}}{k^{\prime}-k} d k^{\prime}, \\
k \text { on } C_{1},
\end{array}
$$

$$
\pi i X_{2}^{+}(k) \phi_{2}(k, \alpha)=\frac{-2(-\alpha+k)^{1 / 2} e^{i \alpha}}{\alpha+k}-\int_{C_{1}} \frac{\phi_{1}\left(k^{\prime}, \alpha\right) X_{2}\left(k^{\prime}\right) e^{-2 i k^{\prime}}}{k^{\prime}-k} d k^{\prime}
$$




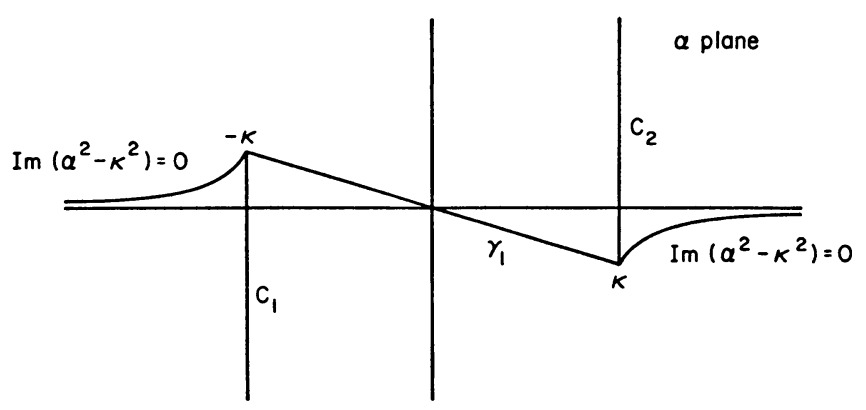

FIGURE 1

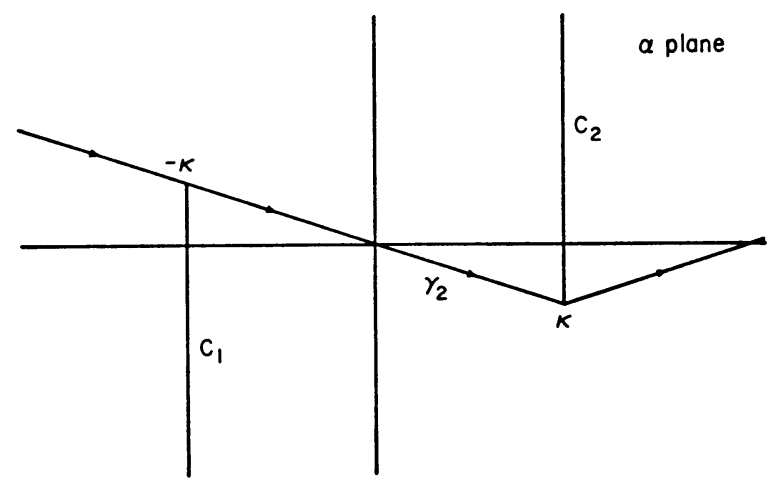

FiguRE 2

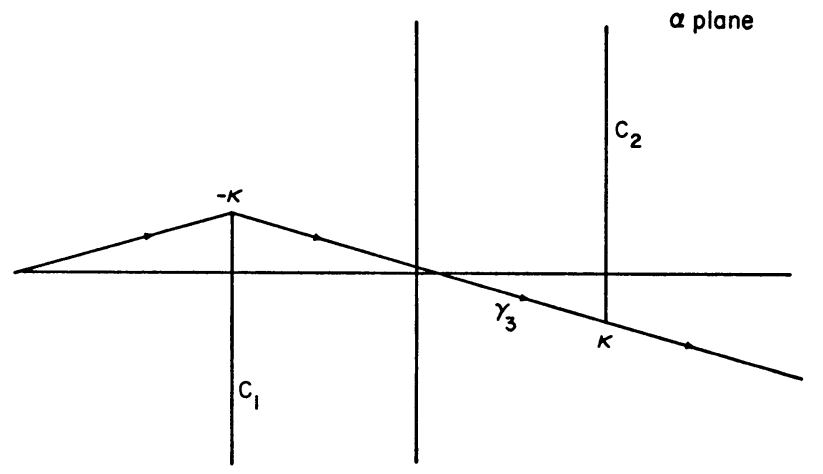

FIGURE 3

This is the solution method of [9]. The contours $C_{1}$ and $C_{2}$ are pictured in Figures 1,2 and 3. The function $(k-\kappa)^{1 / 2}$ is defined in the $k$ plane slit along $C_{2}$ and taken to be positive for $\operatorname{Im} k=\kappa_{2}, \operatorname{Re} k>\kappa_{1}$. The function $(k+\kappa)^{1 / 2}$ is defined in the $k$ plane slit along $C_{1}$ and taken to be positive for $\operatorname{Im} k=-\kappa_{2}$, $\operatorname{Re} k>-\kappa_{1}$. In $(2.4) X_{1}(k)=(k+\kappa)^{-1 / 2}$ and $X_{2}(k)=(k-\kappa)^{-1 / 2}$. For $k$ on 
$C_{1}, X_{1}^{+}(k)$ refers to the values of $X_{1}$ on the left side of the slit $C_{1}$, while for $k$ on $C_{2}, X_{2}^{+}(k)$ refers to the values of $k$ on the right side of the slit $C_{2}$.

As usual these equations are solved by successive approximations. We parameterize $C_{1}$ by letting $k=-\kappa-i s \kappa_{1}, 0 \leqslant s<\infty$, and $C_{2}$ by letting $k=\kappa+i t \kappa_{1}, 0 \leqslant t<\infty$. Let $\phi_{1}\left(-\kappa-i \kappa_{1} s, \alpha\right)=\psi_{1}(s, \alpha)$ and $\phi_{2}\left(\kappa+i t \kappa_{1}, \alpha\right)$ $=\psi_{2}(t, \alpha)$. We also set $\kappa_{2}=-\kappa_{1} \beta$. We will assume that $0 \leqslant \beta \leqslant \frac{1}{2}$. With these substitutions (2.4) becomes

$$
\begin{aligned}
\psi_{1}(s, \alpha)= & \frac{2}{\pi} e^{i \pi / 4} \frac{(-\alpha-\kappa)^{1 / 2} \kappa_{1}^{1 / 2} s^{1 / 2} e^{-i \alpha}}{\alpha+k} \\
& +\frac{e^{3 \pi i / 4}}{\pi} s^{1 / 2} e^{2 i \kappa} \int_{0}^{\infty} \frac{\psi_{2}\left(t^{\prime}, \alpha\right) e^{-2 \kappa_{1} t^{\prime}} d t^{\prime}}{\left(2+i\left(t^{\prime}+s-2 \beta\right)\right)\left(2+i\left(t^{\prime}-2 \beta\right)\right)^{1 / 2}},
\end{aligned}
$$

$$
\begin{aligned}
\psi_{2}(t, \alpha)= & \frac{2}{\pi} e^{3 \pi i / 4} \frac{(-\alpha+\kappa)^{1 / 2} \kappa_{1}^{1 / 2} t^{1 / 2} e^{i \alpha}}{\alpha+k} \\
& +\frac{e^{i \pi / 4}}{\pi} t^{1 / 2} e^{2 i \kappa} \int_{0}^{\infty} \frac{\psi_{1}\left(s^{\prime}, \alpha\right) e^{-2 \kappa_{1} s^{\prime}} d s^{\prime}}{\left(2+i\left(t+s^{\prime}-2 \beta\right)\right)\left(-2-i\left(s^{\prime}-2 \beta\right)\right)^{1 / 2}} .
\end{aligned}
$$

We now specify the contour $\gamma$ over which $\alpha$ runs.

(a) If $y \neq 0$ we take $\gamma=\gamma_{1}$ where $\gamma_{1}$ is the contour shown in Figure 1.

(b) If $y=0, x>1$ we take $\gamma=\gamma_{2}$ where $\gamma_{2}$ is the contour shown in Figure 2.

(c) If $y=0, x<-1$ we take $\gamma=\gamma_{3}$ where $\gamma_{3}$ is the contour shown in Figure 3.

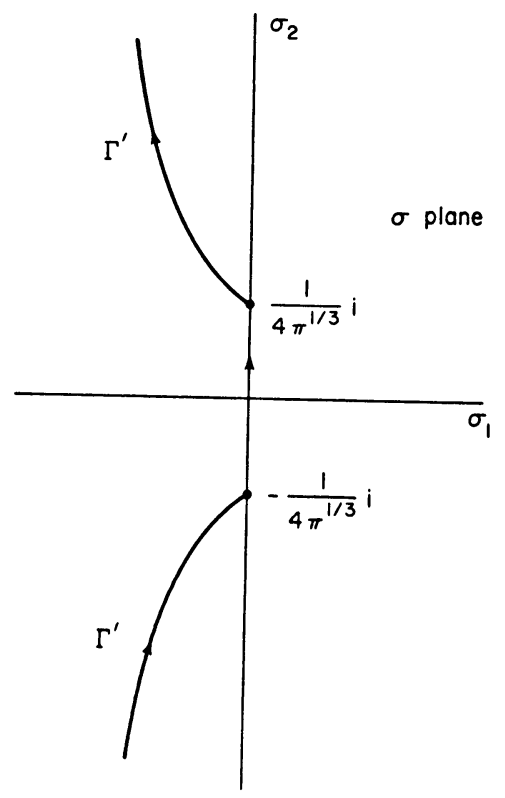

FIGURE 4 
The analysis of equations (2.5) is independent of the position of $(x, y)$. We take the zero approximations $\psi_{1}^{(0)}(s, \alpha)$ and $\psi_{2}^{(0)}(t, \alpha)$ to be the inhomogeneous terms in (2.5). For $\alpha$ on $\gamma$ and $k=-\kappa-i s \kappa_{1}$ on $C_{1}$ we have $|\alpha+k| \geqslant \kappa_{1} s / 2$, while for $\alpha$ on $\gamma$ and $k=\kappa+i t \kappa_{1}$ on $C_{2}$ we have $|\alpha+k| \geqslant \kappa_{1} t / 2$ (in all cases). Hence, if $\alpha=\alpha_{1}+i \alpha_{2}$,

$$
\begin{aligned}
& \left|\psi_{1}^{(0)}(s, \alpha)\right| \leqslant(4 / \pi)\left(\kappa_{1} s\right)^{-1 / 2}|-\alpha-\kappa|^{1 / 2} e^{\alpha_{2}}, \\
& \left|\psi_{2}^{(0)}(t, \alpha)\right| \leqslant(4 / \pi)\left(\kappa_{1} t\right)^{-1 / 2}|-\alpha+\kappa|^{1 / 2} e^{-\alpha_{2}} .
\end{aligned}
$$

Let

$$
M_{\alpha, \kappa}=\max \left\{|-\alpha-\kappa|^{1 / 2} e^{\alpha_{2}},|-\alpha+\kappa|^{1 / 2} e^{-\alpha_{2}}\right\}
$$

Then

$$
\begin{aligned}
\mid \psi_{1}^{(1)}(s, \alpha) & -\psi_{1}^{(0)}(s, \alpha) \mid \\
& \leqslant \frac{4}{\pi^{2}} s^{1 / 2} \kappa_{1}^{-1 / 2} e^{-2 \kappa_{2}} M_{\alpha, \kappa} \int_{0}^{\infty} \frac{t^{\prime-1 / 2} e^{-2 \kappa_{1} t^{\prime}} d t^{\prime}}{\left[4+\left(t^{\prime}-2 \beta\right)^{2}\right]^{1 / 4}\left[4+\left(t^{\prime}+s-2 \beta\right)^{2}\right]^{1 / 2}} \\
& \leqslant \frac{4}{\pi^{2}} s^{1 / 2} \kappa_{1}^{-1 / 2} e^{-2 \kappa_{2}} M_{\alpha, \kappa} g(s) \int_{0}^{\infty} t^{-1 / 2} e^{-2 \kappa_{1} t^{\prime}} d t^{\prime} \\
& =(2 / \pi)^{3 / 2} s^{1 / 2} g(s) e^{-2 \kappa_{2}} \kappa_{1}^{-1} M_{\alpha, \kappa} .
\end{aligned}
$$

Here

$$
g(s)=\left\{\begin{array}{l}
\frac{1}{2}, \quad s \leqslant 2 \beta, \\
\left(4+(s-2 \beta)^{2}\right)^{-1 / 2}, \quad s \geqslant 2 \beta .
\end{array}\right.
$$

An entirely analogous estimate holds for $\left|\psi_{2}^{(1)}(t, \alpha)-\psi_{2}^{(0)}(t, \alpha)\right|$. By induction then

$$
\begin{array}{r}
\left|\psi_{1}^{(n)}(s, \alpha)-\psi_{1}^{(n-1)}(s, \alpha)\right| \leqslant\left(\frac{2}{\pi}\right)^{3 / 2} s^{1 / 2} g(s) M_{\alpha, \kappa} e^{-2 \kappa_{2}} \kappa_{1}^{-1}\left(\frac{e^{-4 \kappa_{2}}}{256 \kappa_{1}^{3}}\right)^{(n-1) / 2}, \\
\left|\psi_{2}^{(n)}(t, \alpha)-\psi_{2}^{(n-1)}(t, \alpha)\right| \leqslant\left(\frac{2}{\pi}\right)^{3 / 2} t^{1 / 2} g(t) M_{\alpha, \kappa} e^{-2 \kappa_{2}} \kappa_{1}^{-1}\left(\frac{e^{-4 \kappa_{2}}}{256 \kappa_{1}^{3}}\right)^{(n-1) / 2}, \\
n=1,2,3, \ldots .
\end{array}
$$

Thus if

$$
e^{-\kappa_{2}}<4 \pi^{1 / 4} \kappa_{1}^{3 / 4}, \quad \kappa_{2} \leqslant 0,
$$


the sequences $\left\{\psi_{1}^{(n)}(s, \alpha)\right\}$ and $\left\{\psi_{2}^{(n)}(t, \alpha)\right\}$ converge uniformly in $s$ and $t$, respectively, for each $\alpha \neq \pm \kappa$ to functions $\psi_{1}(s, \alpha)$ and $\psi_{2}(t, \alpha)$ and this pair of functions satisfies (2.5).

We now suppose

$$
e^{-\kappa_{2}} \leqslant 8^{1 / 2} \pi^{1 / 4} \kappa_{1}^{3 / 4}, \quad \kappa_{2} \leqslant 0
$$

then

$$
\left|\psi_{1}(s, \alpha)\right| \leqslant(4 / \pi)\left(\kappa_{1} s\right)^{-1 / 2} M_{\alpha, \kappa}+2(2 / \pi)^{3 / 2} s^{1 / 2} g(s) \kappa_{1}^{-1} M_{\alpha, \kappa}
$$

with a similar estimate holding for $\psi_{2}(t, \alpha)$.

Since $g(s) \leqslant 2^{1 / 2} / s$ for $s>0$, we obtain

$$
\begin{aligned}
& \left|\psi_{1}(s, \alpha)\right| \leqslant\left(16 / \pi^{3 / 2}\right)\left(\kappa_{1} s\right)^{-1 / 2} M_{\alpha, \kappa}\left(1+\kappa_{1}^{-1 / 2}\right), \\
& \left|\psi_{2}(t, \alpha)\right| \leqslant\left(16 / \pi^{3 / 2}\right)\left(\kappa_{1} t\right)^{-1 / 2} M_{\alpha, \kappa}\left(1+\kappa_{1}^{-1 / 2}\right) .
\end{aligned}
$$

From this we obtain

$$
\begin{aligned}
& \left|\int_{C_{1}} e^{i k(\xi-1)} \phi_{1}(k, \alpha) d k+\int_{C_{2}} e^{i k(\xi+1)} \phi_{2}(k, \alpha) d k\right| \\
& \quad \leqslant(16 / \pi) M_{\alpha, \kappa}\left(1+\kappa_{1}^{-1 / 2}\right)\left\{e^{\kappa_{2}(\xi-1)}(1-\xi)^{-1 / 2}+e^{-\kappa_{2}(\xi+1)}(1+\xi)^{-1 / 2}\right\} .
\end{aligned}
$$

Thus from (2.2), (2.3) and (2.8) we obtain

$$
\begin{aligned}
|\rho(\xi)| \leqslant & 2\left|(\partial / \partial \eta) H_{0}^{(1)}\left(\kappa\left[(x-\xi)^{2}+(y-\eta)^{2}\right]^{1 / 2}\right)\right|_{\eta=0} \mid \\
& +\frac{4}{\pi^{2}}\left(1+\kappa_{1}^{-1 / 2}\right)\left\{e^{\kappa_{2}(\xi-1)}(1-\xi)^{-1 / 2}+e^{-\kappa_{2}(\xi+1)}(1+\xi)^{-1 / 2}\right\} \\
& \times\left|\int_{\gamma} M_{\alpha, \kappa}\left(\alpha^{2}-\kappa^{2}\right)^{-1 / 2} \exp \left\{i \alpha x-\left(\alpha^{2}-\kappa^{2}\right)^{1 / 2}|y|\right\} d \alpha\right| .
\end{aligned}
$$

We now estimate the integral in (2.9). We first assume $y \neq 0$ so that $\gamma=\gamma_{1}$. On that portion of $\gamma_{1}$ lying to the right of the point $\kappa$ we have

$$
\begin{aligned}
M_{\alpha, \kappa}\left|\left(\alpha^{2}-\kappa^{2}\right)\right|^{-1 / 2} & \leqslant e^{-\kappa_{2}}\left(\alpha_{1}-\kappa_{1}\right)^{-1 / 2} ; \\
\left|\exp \left\{i \alpha x-\left(\alpha^{2}-\kappa^{2}\right)^{1 / 2}|y|\right\}\right| & \leqslant \exp \left\{-\alpha_{2}|x|-|y|\left(\alpha_{1}^{2}-\alpha_{2}^{2}-\kappa_{1}^{2}+\kappa_{2}^{2}\right)^{1 / 2}\right\} \\
& \leqslant \exp \left\{-\kappa_{2}|x|-\alpha_{1}|y|+|y|\left(\alpha_{2}^{2}+\kappa_{1}^{2}-\kappa_{2}^{2}\right)^{1 / 2}\right\} \\
& \leqslant \exp \left\{-\kappa_{2}|x|-\alpha_{1}|y|+\kappa_{1}|y|\right\} .
\end{aligned}
$$


Here we use the fact that on the curve in question, $\alpha_{1}^{2} \geqslant \kappa_{1}^{2}+\alpha_{2}^{2}-\kappa_{2}^{2}>0$, and if $A>B>0$, then $\left(A^{2}-B^{2}\right)^{1 / 2}>A-B$. Finally on this portion of the curve $|d \alpha| \leqslant 2 d \alpha_{1}$. Thus this integral is dominated by

$$
2 e^{-\kappa_{2}(|x|+1)} e^{\kappa_{1}|y|} \int_{\kappa_{1}}^{\infty} e^{-|y| \alpha_{1}}\left(\alpha_{1}-\kappa_{1}\right)^{-1 / 2} d \alpha_{1}=2 \pi^{1 / 2}|y|^{-1 / 2} e^{-\kappa_{2}(|x|+1)} .
$$

The same estimate holds for the integral over the part of $\gamma_{1}$ lying to the left of $-\kappa$. On the straight line portion of $\gamma_{1}$ we let $\alpha=\kappa t,-1 \leqslant t \leqslant 1$. Then

$$
\begin{gathered}
M_{\alpha, \kappa}\left|\left(\alpha^{2}-\kappa^{2}\right)\right|^{-1 / 2} \leqslant 2^{1 / 2} \kappa_{1}^{-1 / 2}\left(1-t^{2}\right)^{-1 / 2} e^{-\kappa_{2}}, \\
\left|\exp \left\{i \alpha x-\left(\alpha^{2}-\kappa^{2}\right)^{1 / 2}|y|\right\}\right| \leqslant e^{-\kappa_{2}(|x|+|y|)}, \\
|d \alpha| \leqslant 2^{1 / 2} \kappa_{1} d t .
\end{gathered}
$$

From this we see that the integral is dominated by $2 \pi \kappa_{1}^{1 / 2} e^{-\kappa_{2}(1+|x|+|y|)}$. Thus the integral over $\gamma_{1}$ is dominated by $C_{1}^{1 / 2} e^{-k_{2}(1+|x|+|y|)}$. It then follows from (2.9) that

$$
\begin{aligned}
|\rho(\xi)| \leqslant & 2\left|(\partial / \partial \eta) H_{0}^{(1)}\left(\kappa\left[(x-\xi)^{2}+(y-\eta)^{2}\right]^{1 / 2}\right)\right|_{\eta=0} \mid \\
& +C \kappa_{1}^{1 / 2}\left(1+\kappa_{1}^{-1 / 2}\right) e^{-\kappa_{2}(|x|+|y|+1)} \\
& \times\left\{e^{\kappa_{2}(\xi-1)}(1-\xi)^{-1 / 2}+e^{-\kappa_{2}(\xi+1)}(1+\xi)^{-1 / 2}\right\} .
\end{aligned}
$$

We now return to (2.9) and consider the case where $y=0, x>1$. In this case $\gamma=\gamma_{2}$.

On the portion of $\gamma_{2}$ to the right of $\kappa$,

$$
M_{\alpha, \kappa}\left|\left(\alpha^{2}-\kappa^{2}\right)^{-1 / 2}\right| \leqslant\left(\alpha_{2}-\kappa_{2}\right)^{-1 / 2} e^{\alpha_{2}-2 \kappa_{2}}
$$

so that the integral over that portion of $\gamma_{2}$ is dominated by

$$
2 e^{-2 \kappa_{2}} \int_{\kappa_{2}}^{\infty}\left(\alpha_{2}-\kappa_{2}\right)^{-1 / 2} e^{-\alpha_{2}(x-1)} d \alpha_{2}=2 \pi^{1 / 2}(x-1)^{-1 / 2} e^{-\kappa_{2}(x+1)} .
$$

On the portion of $\gamma_{2}$ to the left of $-\kappa$,

$$
M_{\alpha, \kappa}\left|\left(\alpha^{2}-\kappa^{2}\right)^{-1 / 2}\right| \leqslant\left(\alpha_{2}+\kappa_{2}\right)^{-1 / 2} e^{\alpha_{2}}
$$

so that the integral over that portion of $\gamma_{2}$ is dominated by

$$
2 \int_{-\kappa_{2}}^{\infty}\left(\alpha_{2}+\kappa_{2}\right)^{-1 / 2} e^{-\alpha_{2}(x-1)} d \alpha_{2}=2 \pi^{1 / 2}(x-1)^{-1 / 2} e^{-\kappa_{2}(x-1)} .
$$

The integral over the remaining portion of $\gamma_{2}$ is estimated much as in the first 
case. The result is that the integral is dominated by $2 \pi \kappa_{1}^{1 / 2} e^{-\kappa_{2}(x+1)}$. Thus the integral over $\gamma_{2}$ is dominated by $C \kappa_{1}^{1 / 2} e^{-\kappa_{2}(x+1)}$ and we again obtain the estimate (2.10) (with $y=0$ ).

The case $y=0, x<-1$, in which we take $\gamma=\gamma_{3}$, is handled in the same manner and again we arrive at (2.10) (with $y=0$ ). In (2.10) the constant depends on $x$ and $y$.

An argument in [10] shows that the solution we have found is unique. Thus we have proven

THEOREM 2. The unique solution $\tilde{\rho}(\xi, x, y, \kappa)$ of (2.1) for $\kappa$ satisfying (2.7a) is given by (2.2), (2.3) where $\phi_{1}(k, \alpha), \phi_{2}(k, \alpha)$ are the unique solutions of (2.4). If $\kappa$ satisfies (2.7b), the estimate (2.10) is valid. In (2.10) $C$ depends on $x$ and $y$.

3. Solution of the integral equation in the "low frequency" case. In this section we study the equation

$$
g(s)=\int_{-1}^{1} H_{0}^{(1)}\left(\kappa\left|s-s^{\prime}\right|\right) \rho\left(s^{\prime}\right) d s^{\prime}, \quad|s| \leqslant 1 .
$$

We will show how to solve (3.1) for small positive values of $\kappa$. The method of solution is due to Sologub [6]. In [7] the author introduced the function spaces $L_{2}(q), W_{2}^{1}(q)$ in order to study (3.1). Examination of Sologub's method showed that it fit naturally into the context of these spaces. In [11] it was shown how these ideas could be brought together to solve a related problem. Here we use the Sologub method to investigate how the norm of the solution operator for (3.1) depends on $\kappa$ for small values of $\kappa$. We then apply this result to equation $(0.1)$.

3.1. Preliminaries. (a) Definition of the spaces $L_{2}(q), W_{2}^{1}(q)$ and $\mathbb{Q}$. $L_{2}(q)=\left\{f:\|f\|_{L_{2}(q)}^{2}=\int_{-1}^{1}|f(t)|^{2}\left(1-t^{2}\right)^{1 / 2} d t<\infty\right\}$,

$W_{2}^{1}(q)=\left\{f: f\right.$ is absolutely continuous on $\left.[-1,1], f^{\prime} \in L_{2}(q)\right\}$. We equip $W_{2}^{1}(q)$ with the norm

$$
\begin{gathered}
\|f\|_{W_{2}^{1}(q)}^{2}=\|f\|_{L_{2}(q)}^{2}+\left\|f^{\prime}\right\|_{L_{2}(q)}^{2} . \\
\mathcal{Q}=\left\{\phi: \phi(\lambda)=\frac{1}{2 \pi} \int_{-1}^{1} f(\xi) e^{-i \lambda \xi} d \xi ; f \in L_{2}(q)\right\} .
\end{gathered}
$$

We equip $Q$ with the norm of $L_{2}(q)$.

The right hand side of (3.1) defines an operator $L_{\kappa}$. We have

THEOREM [7]. The operator $L_{\kappa}$ for $\kappa>0$ is a one-to-one bicontinuous map of $L_{2}(q)$ onto $W_{2}^{1}(q)$.

Here we wish to derive an estimate for $\left\|L_{\kappa}^{-1}\right\|$ which is valid for small values of $\kappa$. 
An appiication of Hölder's inequality shows that $L_{2}(q) \hookrightarrow L_{p}(-1,1)$ for $1 \leqslant p<\frac{4}{3}$. Thus if we extend functions in $L_{2}(q)$ by setting them equal to zero for $|t| \geqslant 1$, we have, with $L_{p}=L_{p}(-\infty, \infty)$,

$$
\|f\|_{L_{p}} \leqslant C_{p}\|f\|_{L_{2}(q)} \quad \forall f \in L_{2}(q), 1 \leqslant p<\frac{4}{3} .
$$

In particular we can take $C_{1}=\pi^{1 / 2}$ in (3.2). We also have the inclusions $W_{2}^{1}(q) \hookrightarrow L_{\infty}$. To derive this we let $g \in W_{2}^{1}(q)$; then for $-1 \leqslant x, y \leqslant 1$,

$$
\begin{gathered}
g(x)=g(y)+\int_{y}^{x} g^{\prime}(t) d t, \\
|g(x)| \leqslant|g(y)|+\left|\int_{y}^{x} g^{\prime}(t) d t\right| \leqslant|g(y)|+\left\|g^{\prime}\right\|_{L_{1}} .
\end{gathered}
$$

Integrate with respect to $y$ to obtain

$$
|g(x)| \leqslant \frac{1}{2}\|g\|_{L_{1}}+\left\|g^{\prime}\right\|_{L_{1}} \leqslant \frac{3}{2} C_{1}\|g\|_{W_{2}^{\prime}(q)}
$$

$\left(C_{1}\right.$ as in (3.2)). Hence we find

$$
\|g\|_{L_{\infty}} \leqslant \frac{3}{2} C_{1}\|g\|_{W_{2}^{\prime}(q)} \quad \forall g \in W_{2}^{1}(q) .
$$

Another result we will need concerns singular integral operators acting on $L_{2}(q)$.

THEOREM [7]. Let

$$
(H f)(\tau)=\frac{1}{\pi} \int_{-1}^{1} \frac{f(t) d t}{\tau-t}, \quad|\tau|<1 .
$$

Then $H$ is a continuous map of $L_{2}(q)$ onto $L_{2}(q)$ with a one dimensional nullspace spanned by $p(t)=\left(1-t^{2}\right)^{-1 / 2}$, i.e.,

$$
\int_{-1}^{1}\left(1-t^{2}\right)^{-1 / 2} \frac{d t}{\tau-t}=0, \quad|\tau|<1 .
$$

The map

$$
(T f)(\tau)=\frac{1}{\pi} \frac{1}{\left(1-\tau^{2}\right)^{1 / 2}} \int_{-1}^{1} \frac{f(t)}{t-\tau}\left(1-t^{2}\right)^{1 / 2} d t
$$

is an isometry of $L_{2}(q)$ into $L_{2}(q)$ such that

$$
H T=I .
$$

(b) Fourier transforms on $L_{p}, 1 \leqslant p \leqslant 2$.

It is known [5] that the operator of Fourier transformation $F$ defines a 
bounded linear mapping from $L_{p}$ into $L_{p^{\prime}}$ for $1 \leqslant p \leqslant 2$ and $p^{\prime}=p /(p-1)$. Thus if $f \in L_{p}, 1 \leqslant p \leqslant 2$, and $\hat{f}=F f$,

$$
\|\hat{f}\|_{L_{p^{\prime}}} \leqslant C_{p}^{\prime}\|f\|_{L_{p}} .
$$

From this ensues the embedding $Q \hookrightarrow L_{p^{\prime}}, p^{\prime}>4$, for if $\phi=F(\sigma), \sigma \in L_{2}(q)$, and $1 \leqslant p<\frac{4}{3},\|\phi\|_{L_{p^{\prime}}} \leqslant C_{p}^{\prime}\|\sigma\|_{L_{p}} \leqslant C_{p}^{\prime} C_{p}\|\sigma\|_{L_{2}(q)}=C_{p}^{\prime} C_{p}\|\phi\|_{Q}$. Thus if $\tilde{C}_{p^{\prime}}$ $=C_{p} C_{p}^{\prime}$,

$$
\|\phi\|_{L_{p^{\prime}}} \leqslant \tilde{C}_{p^{\prime}}\|\phi\|_{\mathscr{Q}}, \quad 4<p^{\prime} \leqslant \infty .
$$

An important example of a transform pair is embodied in the formula

$$
H_{0}^{(1)}(\kappa|\xi|)=\frac{1}{\pi} \int_{-\infty}^{\infty} \frac{e^{-i \lambda \xi}}{\left(\kappa^{2}-\lambda^{2}\right)^{1 / 2}} d \lambda .
$$

The function $\left(\kappa^{2}-\lambda^{2}\right)^{1 / 2}$ is defined in the $\lambda$-plane cut along rays going vertically up from $\lambda=\kappa$ and vertically down from $\lambda=-\kappa$ (i.e., $C_{2}$ and $C_{1}$ of $\$ 2$ with $\kappa>0)$ so that its value at $\lambda=0$ is $\kappa$. Thus $\left(\kappa^{2}-\lambda^{2}\right)^{1 / 2} \sim i|\lambda|$ as $|\lambda| \rightarrow \infty$ along the real axis. The function $\left(\kappa^{2}-\lambda^{2}\right)^{-1 / 2}$ is in $L_{p}$ for $1<p<2$. The Parseval relation

$$
\int_{-\infty}^{\infty} \hat{f}(\xi) g(\xi) d \xi=\int_{-\infty}^{\infty} f(\xi) \hat{g}(\xi) d \xi
$$

is valid for all $f$ and $g \in L_{p}, 1 \leqslant p \leqslant 2$, where $\hat{f}=F f, \hat{g}=F g$.

3.2. Solution of the integral equation for small $\kappa$. We seek a solution of (3.1) in $L_{2}(q)$. The function $g$ is in $W_{2}^{1}(q)$. We may differentiate (3.1) to obtain

$$
\begin{aligned}
g^{\prime}(s) & =\int_{-1}^{1} \frac{\partial}{\partial s} H_{0}^{(1)}\left(\kappa\left|s-s^{\prime}\right|\right) \rho\left(s^{\prime}\right) d s^{\prime} \\
& =2 i(H \rho)(s)+\int_{-1}^{1} k\left(s-s^{\prime}\right) \rho\left(s^{\prime}\right) d s^{\prime}, \quad|s|<1,
\end{aligned}
$$

where $H$ is defined by (3.4) and

$$
k(s)=\frac{\partial}{\partial s} H_{0}^{(1)}(\kappa|s|)-\frac{2 i}{\pi} \frac{1}{s} .
$$

The function $k$ is in $L_{p}(-\infty, \infty)$ for $2<p \leqslant \infty$. We now use (3.10) and (3.11) to transform (3.1) and (3.12). Letting $\phi=F \rho(3.1)$ becomes

$$
\frac{1}{2} g(s)=\int_{-\infty}^{\infty} \frac{\phi(\lambda) e^{i \lambda s}}{\left(\kappa^{2}-\lambda^{2}\right)^{1 / 2}} d \lambda, \quad|s|<1,
$$

while (3.12) becomes 


$$
\begin{aligned}
\frac{1}{2} g^{\prime}(s)= & i H F^{-1} \phi(s) \\
& -\int_{-\infty}^{\infty} \phi(\lambda)\left(\frac{-i \lambda}{\left(\kappa^{2}-\lambda^{2}\right)^{1 / 2}}+\frac{|\lambda|}{\lambda}\right) e^{i \lambda s} d \lambda, \quad|s|<1 .
\end{aligned}
$$

We have used Lemma 2 of [11] on the second term on the right of (3.12).

Lemma 3.1. Let $f \in L_{2}(q)$ and $T$ be defined by (3.6). Then for arbitrary complex $C$ the function

$$
\phi(\lambda)=-i F T f(\lambda)+C J_{0}(\lambda)
$$

is in $\mathbb{Q}$ and satisfies

$$
i H F^{-1} \phi(s)=f(s), \quad|s| \leqslant 1 .
$$

Proof. The first term on the right of (3.15) is clearly in $\mathcal{Q} . J_{0} \in \mathbb{Q}$ since

$$
J_{0}(\lambda)=\frac{1}{\pi} \int_{-1}^{1} e^{-i \lambda s}\left(1-s^{2}\right)^{-1 / 2} d s .
$$

Applying $i H F^{-1}$ to $\phi$ and using (3.7) we obtain $i H F^{-1} \phi=f+i C H F^{-1} J_{0}$, but

$$
H F^{-1} J_{0}(s)=\frac{2}{\pi} \int_{-1}^{1}\left(1-s^{2}\right)^{-1 / 2} \frac{d s}{s^{\prime}-s}=0, \quad|s| \leqslant 1,
$$

by (3.5). This proves the lemma.

We define the operator $P$ to be multiplication by the characteristic function of the interval $[-1,1]$ and $\eta(\lambda)=|\lambda| / \lambda-i \lambda /\left(\kappa^{2}-\lambda^{2}\right)^{1 / 2}$. Then $\eta \in L_{p}$ for $1 \leqslant p<2$.

THEOREM 3. Let $\phi \in \mathbb{Q}$ be a solution of the equation

$$
\phi=(1 / i) F T P F^{-1}(\eta \phi)+(1 / 2 i) F T g^{\prime}+C J_{0}
$$

for any C. Then (3.14) is satisfied. Furthermore, if $C$ is chosen so that the corresponding $\phi$ satisfies

$$
\frac{1}{2} g(0)=\int_{-\infty}^{\infty} \frac{\phi(\lambda)}{\left(\kappa^{2}-\lambda^{2}\right)^{1 / 2}} d \lambda,
$$

then $\rho=F^{-1}(\phi)$ is the solution of (3.1).

Proof. Suppose $\phi$ satisfies (3.18). Apply $i H F^{-1}$ to (3.18). Since $\eta \in L_{1}, \eta \phi$ $\in L_{1}$, hence $F^{-1} \eta \phi \in L_{\infty}$ and $P F^{-1} \eta \phi \in L_{2}(q)$ so that $F T P F^{-1}(\eta \phi) \in \mathbb{Q}$. Hence by (3.7) and (3.17) we obtain (3.14). Thus $\phi$ satisfies (3.14) which implies $\rho$ satisfies (3.12). If $\rho$ satisfies (3.12) then 


$$
g(s)+k=\int_{-1}^{1} H_{0}^{(1)}\left(\kappa\left|s-s^{\prime}\right|\right) \rho\left(s^{\prime}\right) d s^{\prime}=2 \int_{-\infty}^{\infty} \frac{\phi(\lambda) e^{i \lambda s}}{\left(\kappa^{2}-\lambda^{2}\right)^{1 / 2}} d \lambda .
$$

If we let $s=0$ and assume (3.19) holds we find $k=0$ so that $\rho$ also satisfies (3.1).

REMARK. (3.18) is equivalent to the equation derived by Sologub.

$$
\phi(\lambda)=\int_{-\infty}^{\infty} \phi(\mu)\left(\frac{-i \mu}{\left(\kappa^{2}-\mu^{2}\right)^{1 / 2}}+\frac{|\mu|}{\mu}\right) V_{\lambda}^{\mu} d \mu+\frac{1}{2} \int_{-\infty}^{\infty} \widehat{g^{\prime}(\mu)} V_{\lambda}^{\mu} d \mu+C J_{0}(\lambda)
$$

where

$$
\begin{aligned}
V_{\lambda}^{\mu} & =\frac{1}{2 \pi^{2} i} \int_{-1}^{1} \frac{e^{-i \lambda t}}{\left(1-t^{2}\right)^{1 / 2}} d t \int_{-1}^{1} \frac{\left(1-\tau^{2}\right)^{1 / 2} e^{i \mu \tau}}{\tau-t} d \tau \\
& =\frac{\lambda}{2(\lambda-\mu)}\left[J_{1}(\lambda) J_{0}(\mu)-J_{0}(\lambda) J_{1}(\mu)\right] .
\end{aligned}
$$

This form of the equation is probably better suited for computations. However, for our purposes form (3.18) is more convenient.

Define the operator $B$ by

$$
B \phi=(1 / i) F T P F^{-1}(\eta \phi) .
$$

As we have seen in the proof of Theorem $1, B$ maps $\mathcal{Q}$ into $\mathcal{Q}$. We wish now to estimate $\|B\|$. Direct computation shows that $\|\eta\|_{L_{1}}=\kappa(\pi+2)$. Thus if $\phi=F \sigma$ (cf. the remark after (3.2)),

$$
\sup |\phi| \leqslant \frac{1}{2 \pi}\|\sigma\|_{L_{1}} \leqslant \frac{1}{2 \pi^{1 / 2}}\|\sigma\|_{L_{2}(q)}=\frac{1}{2 \pi^{1 / 2}}\|\phi\|_{Q} .
$$

Thus $\phi \eta \in L_{1}$ and $\|\phi \eta\|_{L_{1}} \leqslant\left(2 \pi^{1 / 2}\right)^{-1}(\pi+2) \kappa\|\phi\|_{Q}$. It follows that $P F^{-1}(\eta \phi)$ $\in L_{\infty}[-1,1]$ with $\|P F(\eta \phi)\|_{L_{\infty}} \leqslant\left(2 \pi^{1 / 2}\right)^{-1}(\pi+2) \kappa\left\|_{\phi}\right\|_{\mathbb{Q}}$; thus

$$
\left\|P F^{-1}(\eta \phi)\right\|_{L_{2}(q)} \leqslant\left(\frac{\pi}{2}\right)^{1 / 2}\left\|P F^{-1}(\eta \phi)\right\|_{L_{\infty}} \leqslant \frac{1}{2^{3 / 2}}(\pi+2) \kappa\|\phi\|_{Q} .
$$

Finally, recalling that $T$ is an isometry on $L_{2}(q)$ we obtain

$$
\|B \phi\|_{Q} \leqslant\left(2^{3 / 2}\right)^{-1}(\pi+2) \kappa\|\phi\|_{Q} \text {. }
$$

Thus if

$$
\kappa<2^{3 / 2} /(\pi+2)
$$

(3.18) can be solved by iteration to yield a solution of (3.14). 
There remains the problem of choosing $C$ so that (3.19) holds. We assume $\kappa$ satisfies (3.21). Let $\tilde{B}=(I-B)^{-1}$; then the solution of (3.18) is given by

$$
\phi=(2 i)^{-1} \tilde{B} F T g^{\prime}+C \tilde{B} J_{0} .
$$

Inserting this value of $\phi$ in (3.19) we obtain

$$
g(0)=\frac{1}{i} \int_{-\infty}^{\infty} \frac{d \lambda}{\left(\kappa^{2}-\lambda^{2}\right)^{1 / 2}} \tilde{B} F T^{\prime}(\lambda)+2 C \int_{-\infty}^{\infty} \frac{d \lambda}{\left(\kappa^{2}-\lambda^{2}\right)^{1 / 2}} \tilde{B} J_{0}(\lambda)
$$

The integrals on the right in (3.23) are well defined since $\mathscr{Q} \hookrightarrow L_{p^{\prime}}$ for $p^{\prime}>4$ and $\left(\kappa^{2}-\lambda^{2}\right)^{-1 / 2} \in L_{p}$ for $1<p<2$. Later we will estimate these terms using Hölder's inequality with $p=\frac{5}{4}, p^{\prime}=5$. Thus we can solve (3.23) for $C$ if $d \neq 0$ where

$$
d=2 \int_{-\infty}^{\infty} \frac{d \lambda}{\left(\kappa^{2}-\lambda^{2}\right)^{1 / 2}} \tilde{B} J_{0}(\lambda)
$$

So suppose $d=0$. Let $\phi=\tilde{B} J_{0}$ and $\sigma=F^{-1} \phi$. Then $\phi$ satisfies (3.18) with $g^{\prime} \equiv 0$ and hence (3.14) with $g^{\prime} \equiv 0$. Thus $\int_{-1}^{l} H_{0}^{(1)}\left(\kappa\left|s-s^{\prime}\right|\right) \sigma\left(s^{\prime}\right) d s^{\prime}$ $=$ const, and by hypothesis and (3.19) this constant is zero. Thus by uniqueness $\sigma\left(x^{\prime}\right) \equiv 0,\left|x^{\prime}\right|<1$ and so $\phi \equiv 0$. But $\phi=B \phi+J_{0}=J_{0}$ which is a contradiction. Thus $d$ cannot vanish if (3.21) holds and we can use (3.23) to find $C$ and hence $\phi$.

3.3. Estimate of the norm of the solution operator. Denote the operator on the right hand side of (3.1) by $L_{\kappa}$. Then $L_{\kappa}^{-1}$ maps $W_{2}^{1}(q)$ onto $L_{2}(q)$. We now wish to estimate $\left\|L_{\kappa}^{-1}\right\|$ where the norm is taken in $\mathcal{L}\left(W_{2}^{1}(q), L_{2}(q)\right)$.

We start by assuming

$$
\kappa<2^{1 / 2} /(\pi+2)
$$

If (3.25) holds then

$$
\|\tilde{B}\| \leqslant 2
$$

and

$$
\|I-\tilde{B}\| \leqslant\left(2^{1 / 2}\right)^{-1}(\pi+2) \kappa .
$$

We can now estimate $\phi$ as given by (3.22) and (3.23). First consider $d$ given by (3.24). This can be written

$$
d=2 \int_{-\infty}^{\infty} \frac{J_{0}(\lambda)}{\left(\kappa^{2}-\lambda^{2}\right)^{1 / 2}} d \lambda-2 \int_{-\infty}^{\infty} \frac{1}{\left(\kappa^{2}-\lambda^{2}\right)^{1 / 2}}\left[(I-\tilde{B}) J_{0}\right](\lambda) d \lambda
$$


The first term has the value $2 \pi H_{0}^{(1)}(\kappa / 2) J_{0}(\kappa / 2)$ [6]. To estimate the second term we use Hölder's inequality with $p=\frac{5}{4}, p^{\prime}=5$. From (3.9) and (3.27),

$$
\begin{aligned}
\left|\int_{-\infty}^{\infty} \frac{1}{\left(\kappa^{2}-\lambda^{2}\right)^{1 / 2}}\left[(I-\tilde{B}) J_{0}\right](\lambda) d \lambda\right| & \leqslant \tilde{C}_{5}\left\|(I-\tilde{B}) J_{0}\right\|_{\mathbb{Q}}\left\|\left(\kappa^{2}-\lambda^{2}\right)^{-1 / 2}\right\|_{L_{5 / 4}} \\
& \leqslant A \kappa^{4 / 5}
\end{aligned}
$$

where $A$ is a generic constant independent of $\kappa$. Now

$$
H_{0}^{(1)}(\kappa|x|)=(2 i / \pi) \ln (\kappa \gamma|x| / 2 i)|x|+h(x)
$$

with $|h(x)| \leqslant C_{a} \kappa^{2} \ln \kappa,|x| \leqslant a$ (see the discussion after (1.20) for the definition of the logarithm), so that

$$
d=4 i \ln (\kappa \gamma / 4 i)+o(1) \text { as } \kappa \rightarrow 0 .
$$

Now consider the first term on the right in (3.23) which we write as

$$
\frac{1}{i} \int_{-\infty}^{\infty} \frac{d \lambda}{\left(\kappa^{2}-\lambda^{2}\right)^{1 / 2}}\left(F T g^{\prime}\right)(\lambda)+\frac{1}{i} \int_{-\infty}^{\infty} \frac{d \lambda}{\left(\kappa^{2}-\lambda^{2}\right)^{1 / 2}}(I-\tilde{B}) F T g^{\prime}(\lambda) .
$$

Using (3.10) and (3.11) the first term can be written

$$
\begin{aligned}
& \frac{1}{2 i} \int_{-1}^{1} H_{0}^{(1)}(\kappa|t|)\left(T g^{\prime}\right)(t) d t \\
& \quad=\frac{1}{\pi} \ln \kappa \int_{-1}^{1}\left(T g^{\prime}\right)(t) d t+\frac{1}{2 i} \int_{-1}^{1}\left(H_{0}^{(1)}(\kappa|t|)-\frac{2 i}{\pi} \ln \kappa\right)\left(T g^{\prime}\right)(t) d t .
\end{aligned}
$$

By (3.5) and (3.6) the first term is zero. Using the Schwarz inequality in $L_{2}(q)$ the second term is bounded by

$$
\frac{1}{2}\left\|g^{\prime}\right\|_{L_{2}(q)} \int_{-1}^{1}\left(1-t^{2}\right)^{-1 / 2}\left|H_{0}^{(1)}(\kappa|t|)-\frac{2 i}{\pi} \ln \kappa\right|^{2} d t .
$$

The integral can be bounded independently of $\kappa$ for small $\kappa$. Repeating a previous argument

$$
\left|\int_{-\infty}^{\infty} \frac{d \lambda}{\left(\kappa^{2}-\lambda^{2}\right)^{1 / 2}}(I-\tilde{B}) F T g^{\prime}(\lambda)\right| \leqslant A \kappa^{4 / 5}\left\|g^{\prime}\right\|_{L_{2}(q)},
$$

so that

$$
\left|\int_{-\infty}^{\infty} \frac{d \lambda}{\left(\kappa^{2}-\lambda^{2}\right)^{1 / 2}} \tilde{B} F T g^{\prime}(\lambda)\right| \leqslant A\|g\|_{W_{2}^{\prime}(q)} .
$$


From (3.3) $|g(0)| \leqslant(3 / 2) C_{1}\|g\|_{W_{2}^{\prime}(q)}$, so that

$$
C \leqslant d^{-1} A\|g\|_{W_{2}^{1}(q)}
$$

Also

$$
\left\|(2 i)^{-1} \tilde{B} F T g^{\prime}\right\|_{Q} \leqslant\left\|g^{\prime}\right\|_{L_{2}(q)} .
$$

Thus from (3.22) and (3.30) if $\rho$ is the solution of (3.1) and $\phi=F \sigma$,

$$
\|\rho\|_{L_{2}(q)}=\|\phi\|_{\mathcal{Q}} \leqslant d^{-1} A\|g\|_{W_{2}^{\prime}(q)}+\left\|g^{\prime}\right\|_{L_{2}(q)}, \quad 0<\kappa<2^{1 / 2} /(\pi+2) .
$$

The important thing about this estimate is that $A$ is independent of $\kappa$.

3.4. Application to equation (0.1). We now turn our attention back to (0.1). We first observe:

LEMMA 3.2. The solution of the equation

$$
\frac{2 i}{\pi} \int_{-1}^{1} \ln \frac{\kappa \gamma}{2 i}\left|s-s^{\prime}\right| \rho\left(s^{\prime}\right) d s^{\prime}=g(s)
$$

$g \in W_{2}^{1}(q)$, is given by

$$
\begin{aligned}
\rho(s)= & (2 i)^{-1}\left(T g^{\prime}\right)(s) \\
& +\frac{1}{2 i \ln (\kappa \gamma / 4 i)}\left(1-s^{2}\right)^{-1 / 2}\left[g(0)-\frac{1}{\pi} \int_{-1}^{1} \ln |t|\left(T g^{\prime}\right)(t) d t\right] .
\end{aligned}
$$

Proof. The verification of (3.32) is a simple computation. We use the fact

$$
\int_{-1}^{1}\left(1-t^{2}\right)^{-1 / 2} \ln |t-\xi| d t=-\pi \ln 2, \quad|\xi|<1 .
$$

Also from (3.5) and (3.6), if $f \in L_{2}(q)$,

$$
\int_{-1}^{1}(T f)(t) d t=0 .
$$

Thus

$$
\begin{aligned}
\frac{2 i}{\pi} \int_{-1}^{1} \ln \frac{\kappa \gamma}{2 i} \rho\left(s^{\prime}\right) d s^{\prime}=\frac{\ln (\kappa \gamma / 2 i)}{\ln (\kappa \gamma / 4 i)}\left[g(0)-\frac{1}{\pi} \int_{-1}^{1} \ln |t|\left(T g^{\prime}\right)(t) d t\right] \\
\begin{aligned}
\frac{2 i}{\pi} \int_{-1}^{1} \ln \left|s-s^{\prime}\right| \rho\left(s^{\prime}\right) d s^{\prime} & =\frac{1}{\pi} \int_{-1}^{1} \ln \left|s-s^{\prime}\right|\left(T g^{\prime}\right)\left(s^{\prime}\right) d s^{\prime} \\
& -\frac{\ln 2}{\ln (\kappa \gamma / 4 i)}\left[g(0)-\frac{1}{\pi} \int_{-1}^{1} \ln |t|\left(T g^{\prime}\right)(t) d t\right]
\end{aligned}
\end{aligned}
$$


Let $j(s)=\pi^{-1} \int_{-1}^{1} \ln \left|s-s^{\prime}\right|\left(T g^{\prime}\right)\left(s^{\prime}\right) d s^{\prime}$. Then by (3.7),

$$
j^{\prime}(s)=\frac{1}{\pi} \int_{-1}^{1} \frac{1}{s-s^{\prime}}\left(T g^{\prime}\right)\left(s^{\prime}\right) d s^{\prime}=H \operatorname{Tg}^{\prime}(s)=g^{\prime}(s) .
$$

Thus $j(s)=g(s)+C$. Setting $s=0$ gives

$$
C=\frac{1}{\pi} \int_{-1}^{1} \ln |t|\left(T g^{\prime}\right)(t) d t-g(0) .
$$

Hence

$$
\frac{1}{\pi} \int_{-1}^{1} \ln \left|s-s^{\prime}\right|\left(T g^{\prime}\right)\left(s^{\prime}\right) d s^{\prime}=g(s)-\left[g(0)-\frac{1}{\pi} \int_{-1}^{1} \ln |t|\left(T g^{\prime}\right)(t) d t\right] \text {. }
$$

The result now follows from (3.36), (3.37), (3.38).

Using (3.28) we see that if $\rho$ is given by (3.33), then

$$
\left|\int_{-1}^{1} \rho\left(s^{\prime}\right) H_{0}^{(1)}\left(\kappa\left|s-s^{\prime}\right|\right) d s^{\prime}-g(s)\right| \leqslant A \kappa^{2} \ln \kappa\|\rho\|_{L_{2}(q)} .
$$

Note that $\|\rho\|_{L_{2}(q)}$ can easily be estimated in terms of $\|g\|_{W_{2}^{\prime}(q)}$. We now turn to (0.1). Use (3.28) to write

$$
-H_{0}^{(1)}(\kappa R)=-(2 i / \pi) \ln (\kappa \gamma R / 2 i)-h(R)
$$

where $R=\left[(x-s)^{2}+y^{2}\right]^{1 / 2}$ and $h$ is as in (3.28). Let $\rho_{0}$ be defined by (3.33) with $g=-(2 i / \pi) \ln (\kappa \gamma R / 2 i), R^{\prime}=\partial R / \partial s, R_{0}=\left(x^{2}+y^{2}\right)^{1 / 2}$.

$$
\begin{aligned}
\rho_{0}(s)= & -\pi^{-1} T\left(R^{\prime} / R\right)(s) \\
& +\frac{1}{\pi \ln (\kappa \gamma / 4 i)}\left[-\ln \frac{\kappa \gamma}{2 i} R_{0}+\frac{1}{\pi} \int_{1}^{1} \ln |t| T\left(\frac{R^{\prime}}{R}\right)(t) d t\right]\left(1-s^{2}\right)^{-1 / 2} .
\end{aligned}
$$

Observe that $\left\|\rho_{0}\right\|_{L_{2}(q)}$ is bounded independently of $\kappa$. Let $\rho$ be the exact solution of $(0.1)$ and $\rho_{1}=\rho-\rho_{0}$. Then

$$
\begin{aligned}
\int_{-1}^{1} H_{0}^{(1)}\left(\kappa\left|s-s^{\prime}\right|\right) \rho_{1}\left(s^{\prime}\right) d s^{\prime} & =-H_{0}^{(1)}(\kappa R)-\int_{-1}^{1} H_{0}^{(1)}\left(\kappa\left|s-s^{\prime}\right|\right) \rho_{0}\left(s^{\prime}\right) d s^{\prime} \\
& =-h(R)-\int_{-1}^{1} h\left(\left|s-s^{\prime}\right|\right) \rho_{0}\left(s^{\prime}\right) d s^{\prime}=k(\kappa, s) .
\end{aligned}
$$

The function $k$ is analytic in $\kappa$ as an element of $W_{2}^{1}(q)$. Also $\|k(\kappa, \cdot)\|_{W_{2}^{1}(q)}$ $\leqslant C \kappa^{2} \ln \kappa,\|\partial k(\kappa, \cdot) / \partial \kappa\|_{W_{2}^{1}(q)} \leqslant C \kappa \ln \kappa$. Thus applying the basic estimate (3.31),

$$
\left\|\rho_{1}\right\|_{L_{2}(q)} \leqslant C \kappa^{2} \ln \kappa
$$


If we define the Hankel function in the plane slit along the negative real axis then $L_{\kappa}$ is an operator valued analytic function such that $L_{\kappa}^{-1}$ exists for $\kappa>0$. Thus, by a standard theorem on analytic families of operators [1], $L_{\kappa}^{-1}$ is an operator valued analytic function for $\kappa>0$. Thus $\rho_{1}=L_{\kappa}^{-1} k$ is analytic in $\kappa$ for $\kappa>0$ (as an element of $\left.L_{2}(q)\right)$. $\partial \rho_{1} / \partial \kappa$ satisfies the equation

$$
\begin{aligned}
\int_{-1}^{1} H_{0}^{(1)}\left(\kappa\left|s-s^{\prime}\right|\right) & \frac{\partial \rho_{1}}{\partial \kappa}\left(s^{\prime}\right) d s^{\prime} \\
& =-\int_{-1}^{1} \rho_{1}\left(s^{\prime}\right) \frac{\partial}{\partial \kappa} H_{0}^{(1)}\left(\kappa\left|s-s^{\prime}\right|\right) d s^{\prime}+\frac{\partial k}{\partial \kappa}(\kappa, s) .
\end{aligned}
$$

The right hand side can be estimated in the norm of $W_{2}^{1}(q)$ by $C \kappa \ln \kappa$; thus by (3.31),

$$
\left\|\partial \rho_{1} / \partial \kappa\right\|_{L_{2}(q)} \leqslant C \kappa \ln \kappa
$$

\section{REFERENCES}

1. Tosio Kato, Perturbation theory for linear operators, Springer-Verlag, New York, 1966. MR 34 \#3324.

2. P. D. Lax, C. S. Morawetz and R. S. Phillips, Exponential decay of solutions of the wave equation in the exterior of a star-shaped obstacle, Comm. Pure Appl. Math. 16 (1963), 477-486. MR 27 \# 5033.

3. C. S. Morawetz, The limiting amplitude principle, Comm. Pure Appl. Math. 15 (1962), 349-361. MR 27 \#1696.

4. L. A. Muravei, The decrease of solutions of the second exterior boundary-value problem for the wave equation with two space variables, Dokl. Akad. Nauk SSSR 193 (1970), 996-999 = Soviet Math. Dokl. 11 (1970), 1067-1071. MR 42 \#3400.

5. G. Okikiolu, Aspects of the theory of bounded integral operators in $L^{p}$ spaces, Academic Press, New York, 1971.

6. V. G. Sologub, The solution of a certain integral equation of convolution type with finite limits of integration, Ž. Vyčisl. Mat. i Mat. Fiz. 11 (1971), 837-854 = USSR Comput. Math. and Math. Phys. 11 (1971), no. 4, 33-52. MR 45 \#832.

7. Peter Wolfe, On the inverse of an integral operator, Proc. Amer. Math. Soc. 25 (1970), 443-448. MR 41 \#877.

8. - Diffraction of plane waves by a strip; exact and asymptotic solutions, SIAM J. Appl. Math. 23 (1972), 118-132. MR 47 \#626.

9. - Addendum to [8]-the solution in the general case, SIAM J. Appl. Math. 24 (1973), 613-618. MR 47 \#8010.

10. - Diffraction of a pulse by a strip, J. Math. Anal. Appl. 48 (1974), 170-183. MR 50 \#6308.

11. Low frequency diffraction by a hard strip, SIAM J. Appl. Math. 29 (1975),273-287.

Department of Mathematics, University of Maryland, College Park, Maryland 20742 